\title{
INFLUENCE OF THE FDG-PET/CT ON THE DIAGNOSE AND STAGING OF COLORECTAL CANCER
}

\author{
Nikola Y. Kolev, Anton Y. Tonev, Valentin L. Ignatov, Georgi H. Ivanov, Aleksander \\ K. Zlatarov, Georgi Todorov, Krasimir D. Ivanov \\ 1st Clinic of Surgery, University Hospital "St. Marina”, Varna, Bulgaria
}

\begin{abstract}
INTRODUCTION: In patients with colorectal cancer (CRC), preoperative evaluation and staging should focus on techniques that might alter the preoperative or intraoperative surgical plan. Conventional imaging methods (CT, MRI) have low accuracy for identifying the depth of tumour infiltration and have limited ability to detect regional lymph node involvement. The aim of this study was to evaluate the utility of FDG-PET in the initial staging of patients with $\mathrm{CC}$ in comparison with conventional staging methods and to determine its impact on therapeutic management.

METHODS: In First Clinic of Surgery at University Hospital "St. Marina" one hundred and four patients with a diagnosis of CRC (53 males and 51 females; mean age 66.76 12.36 years), selected prospectively. All patients were studied for staging using a standard procedure (CT) and FDG-PET. The reference method was histology. The effect of FDG-PET on the diagnose and the operative treatment was studied.
\end{abstract}

RESULTS: In 14 patients, surgery was contraindicated by FDG-PET owing to the extent of disease (only 6/14 suspected by CT). FDG-PET revealed four synchronous tumours. For $\mathrm{N}$ staging, both procedures showed a relatively high specificity but a low diagnostic accuracy (PET 56\%, CT 60\%) and sensitivity (PET 21\%, CT $25 \%$ ). For $\mathrm{M}$ assessment, diagnostic accuracy was $92 \%$ for FDGPET and $87 \%$ for CT. FDG-PET results led to modification of the therapy approach in $17.85 \%$ of the patients with rectal cancer and in $14.8 \%$ of the patients with colon cancer.

CONCLUSION: Compared with conventional techniques, FDGPET appears to be useful in pre-surgical staging of $\mathrm{CC}$, revealing unsuspected disease and impacting on the treatment approach.

Key words: 18F-FDG, Position emission tomography, Colorectal cancer, Staging.

\section{INTRODUCTION}

Colorectal cancer is the third most frequently diagnosed malignant tumour and the third most frequent cause of cancer death in Western countries. In Spain, it is the second cause of cancer death in both males (after lung cancer) and females (after breast cancer) and represents $11 \%$ of all cancer deaths [1]. The diagnosis of colorectal carcinoma is based on colonoscopy and biopsy. Surgery is the main therapeutic modality for patients with colorectal carcinoma, even in those with metastasis for whom palliative techniques may be beneficial [2]. After initial diagnosis, accurate staging is the next important step in cancer management. Preoperative evaluation and staging should focus on techniques that might preclude surgery entirely, alter the preoperative or intraoperative surgical plan, or indicate the need for preoperative adjuvant therapy [3]. Depth of penetration through the bowel wall, involvement of lymph nodes, and presence of distant organ metastases are prognostic factors in patients with colorectal cancer [2]. Morphological procedures, i.e. computed tomography (CT) and magnetic resonance imaging (MRI), have shown low (although increasing) accuracy for identifying the depth of tumour infiltration within the bowel wall in colon carcinoma and are of limited value in the detection of regional lymph node involvement [2]. Normal-sized lymph nodes may contain tumour, whereas enlarged nodes may merely be reactive. Therefore, for the vast majority of patients with colorectal cancer, a CT or MRI examination is not required for $\mathrm{N}$ staging, which is determined according to surgical and pathological criteria. Nevertheless, most patients undergo a preoperative CT examination of the chest, abdomen and pelvis for detection of metastatic disease [2]. In addition to providing important prognostic information, the identification of distant metastases has been shown to benefit both the initial staging and the follow-up of patients with colorectal cancer [4]. Accurate staging that identifies unsuspected metastatic disease assists in optimising patient management by ruling out surgery in some cases and ensuring an adequate surgical approach in others. The benefits of surgical resection and systemic chemotherapy in prolonging the survival of patients with hepatic metastases have been established in recent years. Outcomes of surgery in patients with resectable liver disease show 5 year survival rates of $40 \%$, compared with no survival at 5 
years in untreated patients $[5,6]$. Current strategies aim to increase the number of candidates for curative hepatic resection. These strategies include the use of preoperative systemic chemotherapy and ablative therapy, which can lead to surgery with curative intent for patients initially thought to have unresectable disease [6-8]. The key element in improving outcome is patient selection, which requires careful assessment of the precise location and extension of hepatic metastases and the exclusion of patients with unresectable extrahepatic disease. The preoperative demonstration of widespread metastatic disease will preclude surgery in some patients, thereby avoiding operative morbidity if the primary tumour is asymptomatic and does not cause obstruction [6]. Fluoro-2-deoxy-Dglucose (FDG) is a glucose analogue that is phosphorylated in the cells but is not further metabolised. Most malignant tumours show increased uptake of FDG, because malignant transformation and tumour cell growth are associated with overexpression of glucose transporters and increased hexokinase activity [9].

Many studies have reported the value of positron emission tomography (PET) with FDG in the assessment of various malignant primary and recurrent tumours $[10,11]$, including recurrent and metastatic colorectal cancers [1012]. In patients with the latter, FDG-PET appears to be more sensitive than ultrasound, CT or MRI for the detection of liver metastases [13], and additional disease sites are identified in up to $23 \%$ of patients [14]. However, the literature on the performance of FDG-PET in the initial staging of colorectal cancer is limited [15-17] and based on a small number of patients, restricting our ability to draw definitive conclusions about the utility of this technique in the staging of $\mathrm{N}, \mathrm{M}$ or even $\mathrm{T}$ (in synchronous tumour cases) status. The aim of this study was to compare the diagnostic accuracy of FDG-PET with that of conventional staging methods in the initial staging of patients with colorectal carcinoma and to determine the repercussions of this technique for therapeutic management.

\section{MATERIALS AND METHODS}

The patients in this study have referred to First Clinic of Surgery at University Hospital "St. Marina" with a histological diagnosis of colorectal carcinoma between May 2008 and September 2010 and were studied following the standardised protocol for staging and establishing the most adequate treatment. This protocol consisted of: (a) clinical anamnesis, (b) haematological analyses, (c) CT of the abdomen and the thorax (d) endorectal ultrasound in cases of cancer of the lower middle third of the rectum, (e) wholebody FDG-PET. All patients were selected for elective surgery with postoperative adjuvant chemotherapy or palliative chemotherapy, depending on the stage of the disease.Patients with rectal tumour underwent radical surgery with total excision of the mesorectum when tumours were located in the middle or lower thirds. Pathological study of the primary tumour and adenopathies was performed on paraffin-embedded sections stained with haematoxylin-eosin. The most relevant impact on the surgical management of these patients derives from the detection of unsuspected tumour spread. This event has a prevalence of $20 \%[6,18]$. FDG-PET/CT would increase the detection sensitivity up to $90 \%$, according to some authors [19].

One hundred and four consecutive patients from the First Clinic of Surgery with a histological diagnosis of colorectal carcinoma were enrolled in this prospective study: 53 males and 51 females aged from 28 to 83 years (mean age 66.76 years).

Exclusion criteria were:

- presence of other tumour disease,

- history of abdominal surgery,

pregnancy orbreast-feeding,

refusal to participate.

At the time of preoperative diagnosis, tumours were located in the rectum $(n=56)$, sigmoid colon $(n=20)$, ascending colon $(n=2)$, transverse colon $(n=6)$, descending colon $(n=4)$, caecum $(n=10)$ and splenic flexure $(n=6)$. The large number of rectal carcinomas may have been caused by the admission of patients from centres that do not offer surgical treatment of this type of tumour.

\section{CT-study}

All CT scans were performed with a Siemens Somatom Double Source spiral scanner after administration of oral and intravenous contrast. Reports were reviewed for the presence of masses in the colon or rectum, regional lymph node involvement, abdominal lymph node involvement, and distant metastasis. Regional lymph nodes of $1 \mathrm{~cm}$ or larger were scored as positive $\mathrm{N}$, and other site abnormalities interpreted as probable metastatic disease were scored as $\mathrm{M}$ disease.

\section{FDG-PET study}

After administration of $370 \mathrm{MBq}$ 18F-FDG wholebody PET-scan was performed. 5-min emission study was performed for each bed position, including the head, chest, abdomen and pelvis; immediately after the emission studies, a 3-min transmission scan was performed for each bed position. Image processing and reconstruction were performed on a SUN computer Workstation (SUN Microsystem, Mountain View, CA, USA). Images were qualitatively analysed and classified as positive or negative for malignancy by the subjective analysis of a single physician with experience of PET, considering the presence of increased (moderate or intense) glucose uptake as positive for malignancy (taking into consideration the normal biodistribution of the tracer). Semi-quantitative 
analysis was simultaneously performed by calculating the standardised uptake value (SUV) of problem areas, using the slice with maximum count density: $\mathrm{SUV}=$ (injected dose corrected by disintegration/activity concentration)/(injected dose/body weight). A maximal SUV $>2.5$ was considered positive for tumour activity. This cutoff point was for guidance purposes only, however, and the definitive report was predominantly based on qualitative analysis.

\section{Data analysis}

The diagnostic validity of the two procedures in $\mathrm{N}$ and $\mathrm{M}$ staging was analysed by comparing the information in the reports of each examination with the reference criteria, solely considering $\mathrm{N} 0-\mathrm{N}+$ and $\mathrm{M} 0-\mathrm{M}+$ categories. FDG-PET and CT findings were confirmed as follows: (a) by pathological study (b) by surgical exploration and biopsy; (c) in the case of extra-abdominal metastases, by pathological study after biopsy or (when pathology was not available) by clinical follow-up for at least 1 year or by CT study after at least 2 months demonstrating lesion growth $(>0.5 \mathrm{~cm})$ in comparison with the initial examination; or (d) in cases of death, by autopsy examination. Pathology or autopsy material was reviewed in 90 out of the 104 patients and clinical findings and subsequent imaging studies were reviewed in 14 out of the 104 patients to create a final TNM staging according to the American Joint Committee on Cancer (TNM staging system) [20]. Statistical analysis was performed using Microsoft Office Excel to determine the sensitivity, specificity, positive and negative predictive values.

\section{RESULTS}

From 104 patients with histologically confirmed colorectal carcinoma after application of the diagnostic protocol contraindications for surgical treatment were found in 14 of them. These patients did not undergo surgical treatment because its application was contraindicated by the extent of the disease (Table 1). Of these patients, four had multiple unresectable hepatic lesions, two showed lymph node involvement, three had both of the aforementioned conditions, two had hepatic and lung metastases, one patient in stage IV had a hepatic metastasis that was only potentially unresectable but refused any type of treatment; one had no findings that contraindicated surgery but showed peritoneal spread at laparotomy and, finally, one had a functional status that was inadequate for surgery despite having a resectable primary lesion. These patients were referred to the Oncology Department for adjuvant or palliative radiotherapy and/or chemotherapy or were referred to the Palliative Care Department. In these 14 patients, CT (6/14) and/or FDG-PET (14/14) showed the presence of extraregional lymphatic spread or distant metastases, which were confirmed by selective biopsies of problem areas in the Department of Image Diagnostics. Histological study of the primary lesions gave the following results: 59 moderately differentiated adenocarcinomas, 31 well-differentiated adenocarcinomas, two poorly differentiated adenocarcinomas, ten moderately differentiated mucinous adenocarcinomas and two well-differentiated mucinous adenocarcinomas.

Tab. 1. Details of patients who did not undergo surgery

\begin{tabular}{|c|c|c|c|c|c|c|c|c|c|}
\hline Pat. & Localisation & $\begin{array}{l}\mathrm{N} \\
(\mathrm{TAC})\end{array}$ & $\begin{array}{l}\mathrm{N} \\
(\mathrm{TAC})\end{array}$ & $\begin{array}{l}\mathrm{M} \\
(\mathrm{TAC})\end{array}$ & $\mathrm{M}(\mathrm{PET})$ & Coment & $\mathrm{a}$ & $\mathrm{b}$ & $\mathrm{c}$ \\
\hline 1 & Sigmoid colom & 0 & 0 & 0 & Multiple liver metastases & & $\mathrm{a}$ & $\mathrm{b}$ & $\mathrm{c}$ \\
\hline 2 & Colon & 0 & 0 & 0 & Multiple liver metastases & & $\mathrm{a}$ & $\mathrm{b}$ & $\mathrm{c}$ \\
\hline 4 & Caecum & 0 & + & 0 & 0 & $\begin{array}{l}\text { Severe ischaemic } \\
\text { heart disease }\end{array}$ & $\mathrm{a}$ & $\mathrm{b}$ & \\
\hline 6 & Sigmoid colom & 0 & 0 & Multiple liver metastases & Multiple liver metastases & & & & \\
\hline 7 & Rectum & + & + & 0 & Resectable liver metastases & $\begin{array}{l}\text { Patient refused } \\
\text { treatment }\end{array}$ & $\mathrm{a}$ & $\mathrm{b}$ & $\mathrm{c}$ \\
\hline 8 & Rectum & + & + & 0 & Retroperitneal limph nodes & & $\mathrm{a}$ & $\mathrm{b}$ & $\mathrm{c}$ \\
\hline 9 & Rectum & 0 & 0 & 0 & 0 & $\begin{array}{l}\text { Laparotomy } \\
\text { peritoneal } \\
\text { carcinomatosis }\end{array}$ & & & \\
\hline 12 & Rectum & + & + & $+($ single resectable $)$ & $\begin{array}{l}\text { Multiple liver metastases } \\
\text { single limph nodes }\end{array}$ & & $\mathrm{a}$ & & $\mathrm{c}$ \\
\hline
\end{tabular}


a Tumour activity undetected by standard diagnostic procedures

b Staging change

c Modification of therapeutic approach

Both CT and FDG-PET showed changes at the level of the primary lesion that were compatible with tumour status. Most primary tumours showed increased FDG uptake, with intense tracer accumulation in 92 cases, moderate accumulation in 11 cases and no significant uptake in one case (small well-differentiated mucinous adenocarcinoma). FDG-PET revealed synchronous tumours in four patients: moderately differentiated adenocarcinoma and several other small lesions in the sigmoid colon; moderately differentiated adenocarcinomas in the sigmoid colon and splenic angle; moderately differentiated adenocarcinomas in the sigmoid colon and transverse colon; and poorly differentiated adenocarcinomas in the rectum and sigmoid colon. These synchronous lesions were not diagnosed by endoscopy because it was impossible to pass through the primary lesion (adult and paediatric endoscope) and they were only suspected in one case on the basis of CT findings and contrast enema study. Lymphatic spread was studied in the 90 patients who underwent surgery (for whom there was therefore a pathology report), evaluating only the presence or absence of involved lymph nodes (i.e. $\mathrm{N} 0$ or $\mathrm{N}+$ ). CT correctly detected the presence/absence of lymph node involvement in 54 patients, with 36 false negative and two false positive results. FDG-PET correctly detected the presence/absence of lymph node involvement in 50 patients, with 38 false negative and 2 false positive results. The diagnostic validity of the two procedures (in a population with a prevalence of locoregional lymph node tumour disease of $53 \%$ ) is reported in Table 2.

Tab. 2. Diagnosis accuracy in $\mathrm{N} 0 / \mathrm{N}+$ staging

\begin{tabular}{lll}
\hline & FDG-PET & CT \\
\hline Sensitivity & $21 \%[11-35 \%]$ & $25 \%[14-40 \%]$ \\
Speciticity & $95 \%[83-99 \%]$ & $100 \%[83-99 \%]$ \\
Overall accuracy & $56 \%[45-66 \%]$ & $60 \%[49-70 \%]$ \\
PPV & $83 \%[51-97 \%]$ & $100 \%[70-99 \%]$ \\
NPV & $51 \%[40-63 \%]$ & $54 \%[42-65 \%]$ \\
\hline
\end{tabular}

Confidence interval [Cl]: 95\%

Tumour prevalence: 53\% [43-64\%]
Both procedures showed a relatively high specificity but a relatively low overall diagnostic accuracy (PET 56\%, CT 60\%) and sensitivity (PET 21\%, CT 25\%). In the M staging, CT plus chest X-ray correctly classified 90 of the 104 patients, with ten false negatives and four false volumetric projection and a hypermetabolic uptake at the left ileac fossa corresponding to a sigma tumour. No other foci were observed that suggested lymph nodes or distant metastases positives, while FDG-PET correctly classified 96 of the patients, with two false negatives and six false positives. The diagnostic accuracy of the procedures (for a population with a prevalence of metastatic disease of 17\%) is reported in Table 3, showing an overall diagnostic accuracy of $92 \%$ for FDG-PET and $87 \%$ for CT plus chest $\mathrm{X}$-ray.

Tab. 3. Diagnosis accuracy in $\mathrm{M} 0 / \mathrm{M}+$ staging

\begin{tabular}{lll}
\hline & FDG-PET & CT \\
\hline Sensitivity & $89 \%[64-98 \%]$ & $44 \%[22-69 \%]$ \\
Speciticity & $93 \%[85-97 \%]$ & $95 \%[88-98 \%]$ \\
Overall accuracy & $92 \%[85-96 \%]$ & $87 \%[78-92 \%]$ \\
PPV & $73 \%[50-88 \%]$ & $67 \%[35-89 \%]$ \\
NPV & $98 \%[91-100 \%]$ & $89 \%[80-94 \%]$ \\
\hline
\end{tabular}

Confidence interval [Cl]: 95\%

Tumour prevalence: $17 \%$ [43-64\%]

The post-surgical histological examination of lymph nodes showed that 45 patients were in stage N0, 29 in stage $\mathrm{N} 1$ and 16 in stage N2. The definitive staging of the 104 patients was as follows: 20 patients in stage I, 22 in stage II, 34 patients in stage III (4 IIIA, 16 IIIB and 14 IIIC) and 28 in stage IV. The contribution of FDG-PET is shown in detail in Tables 1 and 4. 
Tab. 4. Staging changes due to FDG-PET without modification of resectability

\begin{tabular}{|c|c|c|c|c|c|c|c|}
\hline $\mathrm{a}$ & $\mathrm{b}$ & $\mathrm{C}$ & Localisation & $\begin{array}{l}\text { Staging } \\
\text { according } \\
\text { to } \mathrm{CT}\end{array}$ & $\begin{array}{l}\text { Staging } \\
\text { according } \\
\text { to PET }\end{array}$ & Comment & Over-/understaging \\
\hline \multirow[t]{3}{*}{$\mathrm{a}$} & $\mathrm{b}$ & $\mathrm{c}$ & Colon & III & $\mathrm{IV}$ & Reseclable liver metastasis & Overstaging with surgical changes \\
\hline & $\mathrm{b}$ & $\mathrm{c}$ & Rectum & 11 & III & Unsuspected lymph node & \\
\hline & $\mathrm{b}$ & $\mathrm{c}$ & Colon & 11 & III & Unsuspected lymph node & \\
\hline $\mathrm{a}$ & $\mathrm{b}$ & $\mathrm{c}$ & Rectum & 11 & IV & Resectable liver metastasis & \\
\hline $\mathrm{a}$ & $\mathrm{b}$ & $\mathrm{c}$ & Colon & 11 & IV & Resectable liver metastasis & \\
\hline a & $\mathrm{b}$ & $\mathrm{c}$ & Rectum & II & $\mathrm{IV}$ & Resectable liver metastasis & \\
\hline a & $\mathrm{b}$ & & Rectum & IV & 11 & Non-tumoral liver lesinn & Understaging \\
\hline
\end{tabular}

a - Modification of therapeutic approach

$\mathrm{b}$ - Staging change

c - Tumour activity undetected by standard diagnostic procedures

FDG-PET modified the initial therapeutic approach (from surgery to radio/chemotherapy) in seven out of the subgroup of 14 patients who did not undergo surgery. FDGPET revealed pathological deposits undetected by conventional diagnostic methods in $19.2 \%$ of patients (11 non-operated patients, six operated patients and three patients with unsuspected synchronous tumours). FDGPET changed the staging of the disease in $13.46 \%$ of patients (seven non-operated and seven operated). FDG-PET changed the therapeutic approach in $17.85 \%$ of the patients with rectal cancer and $14.8 \%$ of the patients with colon cancer (12/56; five non-operated patients, four operated patients and three patients with a synchronous tumour).

\section{DISCUSSION}

The objective of preoperative staging is to identify the extent of local infiltration, the degree of lymph node involvement and the presence of hepatic or distant metastases. Correct staging allows treatment to be planned, including surgery, palliative chemotherapy or additional treatments such as neoadjuvant radio- or chemotherapy. This prospective study included 104 consecutive patients with colorectal carcinoma undergoing therapy during the study period. CT and PET were both used, in a standardised preoperative diagnostic procedure, and these two modalities were compared. Reference standard was pathological staging in $91.5 \%$ of cases and oncological follow-up protocol including imaging analysis and clinical examination in the remaining $8.5 \%$. In the present paper, colorectal cancer is considered as a single entity, comprised of two different types of cancer, but both requiring further diagnostics and restaging. In the $\mathrm{T}$ staging of the colorectal cancers, the imaging techniques did not allow evaluation of the degree of infiltration of the intestinal wall by the tumour [2], which was determined by intraoperative observation and histopathological study of the specimen. The lack of reference anatomical structures and its limited spatial resolution makes FDG-PET especially inadequate in furnishing precise information for local staging, and it only offers useful data in cases of gross penetration with invasion of adjacent structures. In rectal tumours, only endorectal ultrasound shows good sensitivity and specificity in the detection of muscularis propria and perirectal tissue invasions [21, 22]. In the present study, FDG-PET correctly detected all primary tumours, identifying four synchronous tumours that had not been localised in the preoperative endoscopic study, although it did not provide information on the degree of infiltration of the intestinal wall. FDG-PET studies are not recommended in current clinical practice for the diagnosis of colorectal cancers or to evaluate their infiltration [20]. Nevertheless, the identification of focal uptake of FDG in the colon should not be ignored, regardless of the clinical setting, since it may correspond to a malignant lesion, as in the case of the four synchronous tumours detected in our series. In more than three-quarters of patients with colorectal cancer, the disease is limited to the intestine or to regional pericolic or mesenteric lymph nodes at the time of diagnosis [2]; therefore, most surgery of colorectal cancer is performed with curative intent. $\mathrm{N}$ staging requires the counting of pericolic and mesenteric lymph nodes. In colorectal carcinoma, regional lymph nodes are frequently small and found close to the primary tumour.

Moreover, pericolic nodes are often small, containing a small number of tumour cells that are only observable in immunohistochemical study [2]. The localisation of lymphatic disease is difficult using imaging techniques only. Despite the identification of lymph nodes as small as $2-3 \mathrm{~mm}$ in highresolution spatial images using multidetector-row spiral CT, the reliable detection of metastases to lymph nodes remains impossible, as the detection is related to the size of the nodes [23]. The drawback of morphological images is that it is difficult to distinguish metastatic from reactive nodes even in case of large lymph nodes and to detect small 
micrometastatic. The high frequency of micrometastasis in nodes of normal size is typical for CRC in comparison to other tumors[24]. There is a wide variation in the reported sensitivity of endorectal ultrasound (62-83\%) and CT $(22-73 \%)$ in the detection of lymphatic involvement, and MRI does not have better effect (39-95\%) due to its volumetric limitation of the diagnostic field [25]. In this study, CT and FDG-PET showed equally poor sensitivity in the detection of regional lymph nodes and in the evaluation of the number involved, with values of $21 \%$ for FDG-PET and $25 \%$ for CT. Nevertheless, FDG-PET was appreciably superior to $\mathrm{CT}$ in the identification of metastatic disease, with a sensitivity of $89 \%$ for FDG-PET versus $44 \%$ for CT. These findings are in agreement with reports by other authors [15-17]. The localisation of lymphatic involvement is determined not only by the size of nodes (in relation to the resolution of the scanner) on the metabolic image with FDG but also by the presence of tumour involvement and its cell load. One explanation for the low sensitivity of FDG-PET in the detection of involved lymph nodes may be their proximity to the primary tumour and bladder, in which the radiopharmaceutical physiologically accumulates. The application of urinary catheterization and diuretics may benefit the interpretation of the image, but this is not the subject of the present study. The presence of extraregional lymph node metastases increases the stage of the disease to IV stage. CRC metastasize via the lymphatic system. The haematogenous spread of colorectal carcinoma can also involve (usually step by step) the liver, lungs and, to a lesser degree, bone, brain and other sites [18]. The most relevant contribution of the proposed procedure is the identification of metastatic disease. Hepatic metastases are found in 10$25 \%$ of patients at initial surgery, and $25 \%$ of these are candidates for resection [2]. In the present series, 28 patients $(26.9 \%)$ were in stage IV. CT had a sensitivity of $44 \%$ and a specificity of $95 \%$ in the diagnosis of metastatic disease, and it detected a patient with retroperitoneal lymph node involvement, while FDGPET showed a sensitivity of $89 \%$ and a specificity of $93 \%$ and revealed that six patients had unsuspected extrahepatic metastatic involvement, mainly in the form of abdominal lymph node involvement and lung metastases.

These data are similar to those reported in previous studies [15-17] and confirm the additional value of FDG-PET, compared with $\mathrm{CT}$, in the preoperative assessment of candidates for curative hepatic resection of colorectal metastases [26, 27]. Regardless of the sensitivity and specificity of FDG-PET for the detection of hepatic metastases, it does not provide sufficient anatomical information for adequate surgical planning. Besides the presence or not of metastasis, knowledge of its precise location is required to plan the appropriate surgical approach or even to exclude surgery. In this context, CT plays a complementary role in the evaluation of these patients. It is expected that new hybrid PET-CT equipment will substantially change this situation, with considerable improvements in diagnostic validity compared with that offered by separate FDG-PET and CT examinations [28]. However, a surgical approach to hepatic disease requires extrahepatic metastatic disease to be ruled out, and it is here that FDG-PET shows its greatest value. In the present series, extrahepatic metastatic sites not detected by other techniques were found in $6.73 \%$ of the patients, leading to a change in the therapeutic strategy. There was a change in therapeutic approach in $25 \%$ of the patients with potentially resectable hepatic metastases. Various studies have also assessed the additional value of FDG-PET compared with CT in these patients, finding that PET offers more information over the provided by $\mathrm{CT}$ and that this information often affects the

treatment. Some authors found that when FDG-PET is additional method to $\mathrm{CT}$ in the preoperative planning of patients with hepatic metastases as additional extrahepatic disease sites were identified in $11-23 \%$ of cases [14, 29, 30]. Identification of such sites frequently leads to a change in approach from surgery to systemic chemotherapy. The sensitivity and specificity of FDG-PET for the detection of disease can be affected by various factors. False positive results have been reported in inflammation settings, such as hepatic abscesses, diverticulitis and active granulomatous disease [22]. Physiological activity in the normal intestine and genitourinary system can also hamper the correct interpretation of certain uptake areas. According to our experience, it is desirable to perform bladder catheterisation and to administer diuretics before the examination in order to avoid accumulation of urine, containing the radiopharmaceutical, in the bladder. False negatives can be produced by tumours with low metabolic activity such as mucinous adenocarcinomas, or by small lesions, micrometastatic disease [22] or the presence of pathological lymph nodes in the immediate vicinity of the primary tumour. The correct anatomical localisation of 18F-FDG deposits can improve their interpretation, and it is hoped that new PETCT scanners, permitting the recording of both anatomical and metabolic information, will enhance the reliability of imaging studies [22]. In this study, FDG-PET changed the tumour staging in $13.46 \%$ of patients, modified the scope of surgery in $11.54 \%$ and led to a change in the therapeutic approach in $17.85 \%$ of those patients with colorectal cancer. These changes were largely based on the diagnosis of previously unsuspected tumour activity, i.e. of synchronous tumours and hepatic and extrahepatic metastases that had not been detected by conventional staging studies. Therefore, FDGPET appears to be highly useful in patients with evidence of resectable hepatic metastasis according to the stage, provided by conventional techniques, and of less utility in the remainder of the patients. Cost-efficiency and cost-efficacy studies, the criteria for selection of patients and the influence of this method on the survivability in the different stages of the disease. 


\section{REFERENCES:}

1. Abdel Nabi H, Doerr RJ, Lamonica DM, Cronin VR, Galantowicz PJ, Carbone GM, et al. Staging of primary colorectal carcinomas with fluorine-18 fluorodeoxyglucose whole-body PET: correlation with histopathologic and CT findings. Radiology 1998; 206:755-60.

2. Skibber JM, Minsky BD, Hoff PM. Cancer of the colon. In: DeVita VT, Hellman S, Rosenberg SA, editors. Cancer. Principles and practice of oncology. Philadelphia: Lippincott, Williams \&Wilkins; 2001; p. 1216-71.

3. Cohen AM. Preoperative evaluation of patients with primary colon cancer. Cancer 1992;70:1328-72.

4. Bleeker WA, Mulder NH, Hermans J, Otter R, Plukker JT. Valueand cost of follow-up after adjuvant treatment of patients withDukes' $\mathrm{C}$ colonic cancer. $\mathrm{Br}$ J Surg 2001; 88:101-6.

5. Geoghegans JG, Scheele J. Treatment of colorectal liver metastases. Br J Surg 1999; 86:158-69.

6. Brown G, Husband JE, Cook G. Colorectal cancer. In: Husband JE, Reznek $\mathrm{RH}$, editors. Imaging in oncology. London: Taylor \& Francis; 2004; p. 217-43.

7. Adam R, Avisar E, Ariche A, Giachetti S, Azoulay D, Castaing D, et al. Five-year survival following hepatic resection alter neoadjuvant therapy for nonresectable colorectal. Ann Surg Oncol 2001;8:347-53.

8. Clavien PA, Selzner N, Morse M, Selzner M, Paulson E. Downstaging of hepatocellular carcinoma and liver metastases 866 Eur J Nucl Med Mol Imaging (2007) 34:859-867 from colorectal cancer by selective intra-arterial chemotherapy. Surgery 2002; 131:433-42.

9. Cook GJR. Artifacts and normal variants in whole-body PET imaging. In: Valk PE, Bailey DL, Townsend DW, Maisey MN, editors. Positron emission tomography. Basic science and clinical practice. London: Springer; 2003; p. 495505.

10. Cabrera Villegas A, Gamez Cenzano C, Martin Urreta JC. Tomografнa por emisiyn de positrones (PET) en oncologна clinica. Rev Esp Med Nuclear 2002;2:131-47.

11. Rodrhguez Fernбndez A, Gymez Rно M, Llamas-Elvira JM, Ortega-Lozano
S, Ferron-Orihuela JA, Ramia-Angel JM, et al. Positronemission tomography with fluorine-18-fluoro-2-deoxy-D-glucose for gallbladder cancer diagnosis. Am J Surg 2004;188:171-5.

12. Tanaka T, Kawai Y, Kanai M, Taki Y, Nakamoto Y, Takabayashi A. Usefulness of FDG-positron emission tomography in diagnosing peritoneal recurrence of colorectal cancer. Am J Surg 2002;184:433-6.

13. Kinkel K, Lu Y, Both M, Warren RS, Thoeni R. Detection of hepatic metastases from cancers of the gastrointestinal tract by using non-invasive imaging methods (US, CT, MR Imaging, PET): a meta-analysis. Radiology 2002;224:748-56.

14. Huebner RH, Park KC, Shepherd JE, Schwimmer J, Czernin J, Phelps ME, et al. A meta-analysis of the literature for whole-body FDG PET detection of recurrent colorectal cancer. J Nucl Med 2000;41:1177-89.

15. Abdel Nabi H, Doerr RJ, Lamonica DM, Cronin VR, Galantowicz PJ, Carbone GM, et al. Staging of primary colorectal carcinomas with fluorine-18 fluorodeoxyglucose whole-body PET: correlation with histopathologic and CT findings. Radiology 1998;206:755-60.

16. Mukai M, Sadahiro S, Yasuda S, Ishida H, Tokunaga N, Tajima $\mathrm{T}$, et al. Preoperative evaluation by whole-body 18F-fluorodeoxyglucose positron emission tomography in patients with primary colorectal cancer. Oncol Rep 2000;7:85-7.

17. Kantorova I, Lipska L, Belohlavek O, Visokai V, Trubac M,Schneiderova M. Routine 18F-FDG-PET preoperative staging of colorectal cancer: comparison with conventional staging and its impact on treatment decision making. J Nucl Med 2003;44:1784-8.

18. Debois JM. TxNxM1: The anatomy and clinics of metastastic cancer. Part II: The primary and its metastases. Boston: Kluwer Academic; 2002; p. 36697.

19. Bipat S, van Leeuwen MS, Comans EFI, Pijl ME, Bossuyt PM, Zwinderman $\mathrm{AH}$, et al. Colorectal liver metastases: CT, MR imaging and PET for diagnosis - metaanalysis. Radiology 2005;237:123-31.

20. American Joint Committee on
Cancer. AJCC Cancer Staging Manual, 6th edition. Berlin Heidelberg, New York: SPringer; 2002; p. 113-23.

21. Bipat S, Glas AS, Slors FJM, Zwinderman AH, Bossuyt PM, Stoker J. Rectal cancer: local staging and assessment of lymph node involvement with endoluminal US, CT, and MR imaging - a meta-analysis. Radiology 2004; 232:773-83.

22. Delbeke D, Martin WH. PET and PET-CT for evaluation of colorectal carcinoma. Semin Nucl Med 2004; 34:209_ 33.

23. Carrington BM. Lymph node metastases. In: Husband JES, Reznek RH, editors. Imaging in oncology. London: Taylor \& Francis; 2004; p. 999-1022.

24. Bjelovic M, Kalezic V, Petrovic M, Pesko P, Usaj SK, Marinkovic J, et al. Correlation of macroscopic and histological characteristics in the regional lymph nodes of patients with rectal and sigmoidal adenocarcinoma. Hepatogastroenterology 1998;45:433-8.

25. Beets-Tan RGH, Beets GL. Rectal cancer: review with emphasis on MR imaging. Radiology 2004; 232:335-46.

26. Vitola JV, Delbeke D, Sandler MP, Campbell MG, Powers TA, Wright JK, et al. Positron emission tomography to stage suspected metastatic colorectal carcinoma to the liver. Am J Surg 1996;171:21-6.

27. Boykin KN, Zibari GB, Lilien DL, McMillan RW, Aultman DF, McDonald JC. The use of FDG-positron emission tomography for the evaluation of colorectal metastases to the liver. Am Surg 1999;65:1183-5.

28. Rohren EM, Turkington TG, Coleman RE. Clinical applications of PET in oncology. Radiology 2004; 231:305-32.

29. Strasberg SM, Dehdashti F, Siegel BA, Drebin JA, Linehan D. Survival of patients evaluated by FDG-PET before hepatic resection for metastatic colorectal carcinoma: a prospective database study. Ann Surg 2001; 233:293-9.

30. Rohren EM, Paulson EK, Hagge R, Wong TZ, Killius J, Clavien PA, et al. The role of $18 \mathrm{~F}-\mathrm{FDG}$ PET in preoperative assessment of the liver in patients being considered for curative resection of hepatic metastases from colorectal cancer. Clin Nucl Med 2002; 27:550-556

\section{Corresponding author:}

Anton Tonev

1st Clinic of Surgery, University Hospital "St. Marina", 1, Hristo Smirnensky str., Varna, 9000, Bulgaria; E-mail: teraton@abv.bg 